This document is published in:

2012 12th IEEE International Conference on Advanced Learning Technologies (ICALT 2012), Rome, Italy, 4-6 July 2012. IEEE, pp. 599-603.

DOI: http://dx.doi.org/10.1109/ICALT.2012.171

(C) 2012 IEEE. Personal use of this material is permitted. Permission from IEEE must be obtained for all other uses, in any current or future media, including reprinting/republishing this material for advertising or promotional purposes, creating new collective works, for resale or redistribution to servers or lists, or reuse of any copyrighted component of this work in other works. 


\title{
Towards efficient provision of feedback supported by learning analytics
}

\author{
Israel Gutiérrez Rojas, Raquel M. Crespo García \\ Dept. Ingeniería Telemática, Universidad Carlos III de Madrid \\ Leganés (Madrid), Spain \\ \{igrojas, rcrespo\}@it.uc3m.es
}

\begin{abstract}
Problem-based learning lab sessions shape a demanding environment, both for students as well for the teaching staff, due to the additional support required. This applies particularly to overcrowded classes. Under these conditions, some aspects do not perform well, like the efficiency of the provision of feedback and the orchestration of the session, jeopardizing the effectiveness of the learning activity. Based on empirical observation, a characterization of lab sessions has been carried out, integrating both qualitative and quantitative parameters describing the interactions that take place. Based on such characterization, a supporting tool is proposed to make use of the students' logs, learning analytics and visualization techniques for providing monitoring and awareness mechanisms for leveraging the detected problems and thus improving the learning and assessment processes.
\end{abstract}

Keywords- Learning analytics; awareness; formative feedback; learning orchestration; assessment.

\section{INTRODUCTION}

Collaborative problem-based learning (henceforth PBL), usually considered an active learning methodology [1], is an instructional method commonly used in engineering courses: students are organized in groups and posed a challenging problem to solve. This approach shapes a more demanding environment, both for students -who require more support from the teaching staff- as well for the teaching staff, particularly in overcrowded classes, i.e., lab sessions with more than 20 students per teacher. Under these conditions, some aspects do not perform well, like the efficiency of the provision of feedback and the orchestration of the session.

In this paper, interactions between teacher and students are observed during a series of typical lab sessions. A set of metrics are thus proposed for characterizing the interaction dynamics, which intends to serve as a comparison framework for efficiency evaluation.

This detailed characterization has been used as a basis for designing a supporting tool, Class-on ${ }^{1}$, aimed at leveraging the detected problems and thus improving the learning and assessment processes. Making use of the students' traces [2], learning analytics and visualization techniques, it is expected to improve the efficiency of the sessions in several aspects:

- Teachers' time management in class (to fairly distribute support and feedback among the students);

- Improving awareness for students (e.g. feedback about their progress related to the average) and for teachers (e.g. session progress in real time);
- Suggesting feedback provision strategies (based on parameters like waiting time, students' progress and risk of fail, supporting time already consumed, etc.);

- Collecting evidences for summative assessment; and

- Analyzing the session for improving future enactments of the learning design.

In a nutshell, based on empirical evidences a tool has being designed that is expected to improve the efficiency of the provision of feedback to the students in lab sessions. Although its experimental validation is not yet completed, empirical data from two different courses supporting its foundation principles are provided.

This paper is organized as follows. The present section introduces the context, goals and hypothesis supporting this work. Section II summarizes related work, including pedagogical and technological basis as well as related projects. Empirical observations of interaction dynamics in lab sessions are discussed in Sections III and a set of metrics for characterizing them are presented in Section IV, for a sound understanding of the requirements. Recommendations for boosting the efficiency of feedback provision in lab sessions are discussed in Section V. Finally, concluding remarks and future work are discussed in Section VI.

\section{RELATED WORK}

As this work focuses on improving learning processes associated to lab sessions by supporting teacher's interventions, formative feedback provision and support for the students based on learning analytics, both pedagogical and technological backgrounds should be considered. In particular, three main research lines are discussed:

- Instructional methodologies: active learning, PBL and formative assessment

- Orchestration and awareness

- Learning analytics for assessment

Regarding active learning, Prince [1] collects use cases in the context of engineering education, but concludes it is the most difficult to analyze since the implementations are very heterogeneous. The use cases of this methodology can emphasize different elements, but the common consequences are that even though the students do not get better assessment results, they are more engaged in the course and, if trained correctly, could develop skills like information retention, problem solving and critical thinking. In [3], Barrows stands out four educational objectives possible with PBL: (a) the knowledge about the context; (b) the practice and feedback; (c) self-directed skills; and (d) motivation and challenge.

${ }^{1}$ www.class-on.org 
In relation to formative assessment, Sadler [4] introduces the importance of qualitative feedback, and highlights the need for self-evaluation during the process. The "assessment as learning" paradigm is reviewed in [5], identifying "assessment as learning" with "self-assessment", i.e. the learner assesses her own work, promoting reflection. A more radical approach is followed in [6], identifying "assessment as learning" with assessment dominating the learning experience, i.e., learning processes consisting only of a continuous assessment/practice. The importance of formative assessment as an awareness mechanism for students [7] and teachers [8], and the orchestration of these mechanisms is generally accepted.

A conceptual framework for orchestration is proposed by Prieto et al. [9], based on a literature review. This framework consists of $5+3$ aspects, one of them being awareness/ assessment. But it is important to highlight that orchestrating learning is not just designing a learning script, but it also has components at run-time when enacting it. This work relates more to this aspect of the orchestration that has to be done at enactment, when the teacher-students interactions take place.

The work by Alavi et al. [10] about distributed awareness mechanisms for orchestration is one of the most relevant for this work and, actually, inspired part of it. It analyzed the interactions between students and teacher at group level from the orchestration of CSCL point of view. Whereas this work is based on a similar approach, there are also key differences: Alavi's focus is on face-to-face sessions, but in this work we explore the orchestration in a blended scenario (and actually, the system could be re-purposed for distance learning), that could combine synchronous and asynchronous interactions (although mainly stresses the former). Other important difference is that, even though both works claim the importance of the space factor in face-to-face situations, Alavi's stressed aspects like human computer interaction and ambient displays; instead, our work stresses the importance of recording the interactions and usage of learning analytics.

Finally, regarding learning analytics for assessment, Blikstein [11] reports its use for collecting evidences for summative assessment. When the students were working on a programming activity, several interactions were collected and analyzed, obtaining conclusions about the strategies followed by the students to construct the programs and about their programming styles. In this work, the idea of collecting evidences for summative assessment is also applied, using the students' progress and eventual delivery of artifacts in order to assess their performance in the session.

\section{EMPIRICAL OBSERVATION OF DYNAMICS IN THE LAB}

\section{A. Context Description}

Eight sessions were observed (14 hours in total), corresponding to Engineering courses ranging from sophomore to senior, in degrees on Communication Systems, Telecommunications, Telematics, and Audiovisual Systems Engineering, covering the most typical cases. They all were practical (hands-on) sessions in a lab, where small groups (24) of students worked with computers; the number of students per session were greater than 20, occasionally greater than 30. Due to administrative restrictions, classes are mostly taught by one unique teacher and only exceptionally reinforced with an additional one for most crowded groups. While the methodology applied is PBL, some of the problem statements are more scripted while others are left more open, depending on the level of the course and the authoring teacher. For completeness, some sessions with summative assessment activities are also considered.

\section{B. Methodology}

Objective and subjective data, covering quantitative and qualitative indicators, were combined for better accuracy.

Objective data were collected by means of empirical observation by an external, neutral observer, unrelated to the course. The following indicators were registered: timestamp of students' help requests; timestamp when the teacher helped them; time devoted by the teacher to each group; order in which feedback was provided to the students; and free text annotations regarding the dynamics in the class.

Subjective data were gathered using students and teachers surveys, questioning about:

- $\quad$ \#questions students made (tutoring help requested)

- Question solving mechanism: teacher's individual/ general explanation, peer's help, or by themselves

- Fairness on the time devoted to each group

- Fairness on the order followed by the teacher to solve questions

- Effectiveness of general explanations by the teacher to the whole audience

- Students' progress during the session

- Open text for additional issues to comment

\section{Experimental Results}

According to teachers' feedback, they often feel overwhelmed, having to deal with more students than they could handle in lab sessions. In general, students devote more attention to individual explanations than to general ones, independently of the delivery time. Frequently, they ask questions that could be easily solved reading the problem statement or the preparing material. When the students' progress is being graded, they require their doubts to be solved as soon as possible. When teachers are aware of several students waiting for help, they try to shorten the explanations aiming to support all of them quickly and fairly. In summary, teachers need awareness on time management in the session; it is even more critical if they have to perform additional tasks (apart from providing feedback), like collecting assessment evidences or delivering specific hardware.

On the other hand, students reflect that they ask too many questions, without trying to solve or thinking by themselves. Several students complain about the difficulty of the proposed problem, stating that reading the preparatory material is not enough to face the problem; some of them indicate that it is too much material to study by themselves, and, before the session, they would need a teacher to explain the proposed exercises and get some feedback about how they tried to solve them. Also, several students think that their lack of previous knowledge (i.e., have not studied the theoretical principles) prevented the teacher of helping them. 
In general, students consider that more teachers are needed (or longer sessions), at least for summative sessions counting for the final grade. They also remark that, if the proposed practice is too scripted, once they got a problem they get stuck and cannot progress without help from the teacher. On a positive note, they report the satisfactory feeling got when they complete the proposed work during the session.

From the perspective of a neutral independent observer, it can be stated that students do not pay enough attention to general explanations longer than 5 minutes. After an individual explanation requiring much time (more than 5 minutes), a lot of new questions are accumulated to be solved. When many doubts rise simultaneously (students with raised hand), teachers tend to help groups nearer to them rather than remember who raised hand first. As the waiting time increases, students get nervous, even standing up and going to the teachers themselves to get attended immediately (when they wait for more than 5 minutes). Finally, regarding summative assessment, the help provided by the teachers in such cases is not so straightforward but they give students some hints to solve the problem instead.

Finally, two interesting conclusions should be remarked based on the analysis of quantitative data. As expected, teachers indicate as the most relevant metrics to distribute their help (in this order): students waiting time, students' progress and tutoring time already devoted to the group. Surprisingly, comparing the data collected from the surveys to the observed data shows that teachers are aware of neither the number of solved doubts, nor the time required for solving them, nor the time the students have been waiting. This is particularly critical as these factors are reported as key for organizing and distributing tutoring help. Probably, the explanation for the discrepancies found is that teachers' attention is focused on the students and their supporting task; thus, they tend to ignore routine tasks and do not keep track of their own time. A supporting tool improving teachers' awareness is consequently expected to be most useful.

\section{PROPOSED METRICS FOR CHARACTERIZATION OF CLASS INTERACTION DYNAMICS}

Both the criteria for distributing the help as well as the metrics used for evaluation or self-reflection are based on basic measures already considered for the study: students' waiting time $\left(t_{w}\right)$, tutoring time $\left(t_{t}\right)$, and students' progress. Based on these primary measures, a set of derived metrics has been defined related to the efficiency of the interactions.

First, aggregated values for each of the primary variables are considered, in order to provide a characterization of the global class rather than the individual students. The total waiting time $\left(\mathrm{T}_{\mathrm{W}}\right)$ in a session is the result of aggregating the waiting time of all the groups of students during the lab session. Similarly, the total tutoring time $\left(\mathrm{T}_{\mathrm{T}}\right)$ is the result of aggregating the tutoring time periods during which the teacher provided feedback to any group. Being $\mathrm{N}$ the number of requests for help, $t_{w i}$ each of the corresponding waiting times and $t_{\mathrm{ti}}$ each of the corresponding tutoring times:

$$
T_{W}=\sum_{i=1}^{N} t_{w i} ; \quad T_{T}=\sum_{i=1}^{N} t_{t i}
$$

Also, both individual and aggregated indicators can be combined to get a normalized metric. The waiting factor $(\mathrm{w})$ is thus defined as the quotient of the waiting time and the tutoring time -either for an individual group (w) or for the global class (W)-, providing a fair insight on the efficiency of the teaching activity (good for values inferior than 1 and hinting to efficiency issues for values greater than 2 ):

$$
w=\frac{t_{w}}{t_{t}} \text { (individual group); } W=\frac{T_{W}}{T_{T}} \text { (global class) }
$$

Finally, metrics regarding the order of the events are also considered. The tutoring order $\left(\mathrm{O}_{\mathrm{T}}\right)$ is defined as the ordered sequence of groups that corresponds to the order followed by the teacher to give feedback to the students:

$$
O_{T}=\left\{G_{1}, G_{2} \ldots G_{N}\right\}
$$

The observed tutoring order $\left(\mathrm{OO}_{\mathrm{T}}\right)$ actually followed by the teacher can be compared to the ideal tutoring order $\left(\mathrm{IO}_{\mathrm{T}}\right)$ to minimize the waiting time. Scheduling algorithms could be applied for improving the efficiency of the sessions, depending on the parameter to optimize (e.g., minimize the total waiting time). For example, First Come First Served, Shortest Job First or Round-Robin.

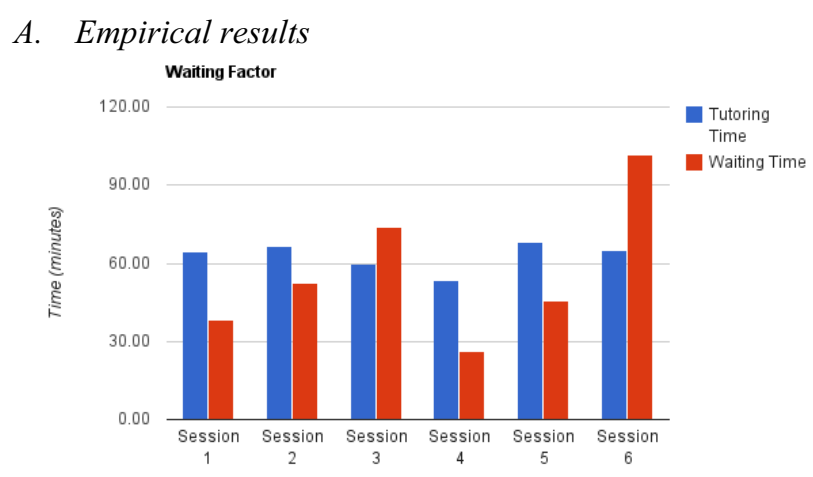

Figure 1. Tutoring time $\left(\mathrm{T}_{\mathrm{T}}\right)$ and waiting time $\left(\mathrm{T}_{\mathrm{W}}\right)$ per session

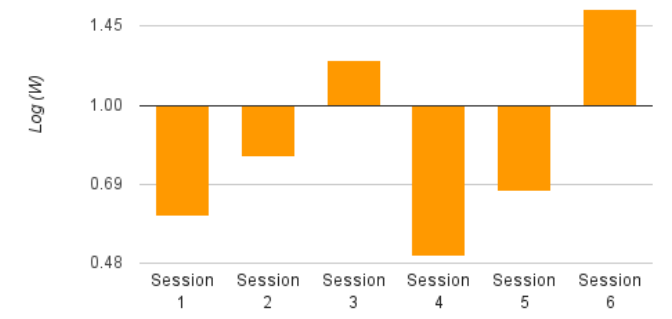

Figure 2. Waiting factor (W) per session (in logarithmic scale)

As observed in Figure 1., the tutoring time is higher than the waiting time in 4 out of 6 observed sessions, hinting to efficient tutoring activity. This is strengthened by Figure 2. , which represents the waiting factor in a log scale in order to emphasize the difference between efficient and non-efficient sessions.

Although only sessions 3 and 6 point to efficiency issues according to the aggregate indicators, a deeper analysis considering the individual metrics provides a more accurate picture. Figure 3. represents the waiting factor of each group and session, showing unfairness problems in the distribution 
of tutoring help. For example, although session 4 was highly efficient according to the aggregate metrics, at least 3 groups appear to have waiting factors above 1, which makes the feedback distribution unfair.

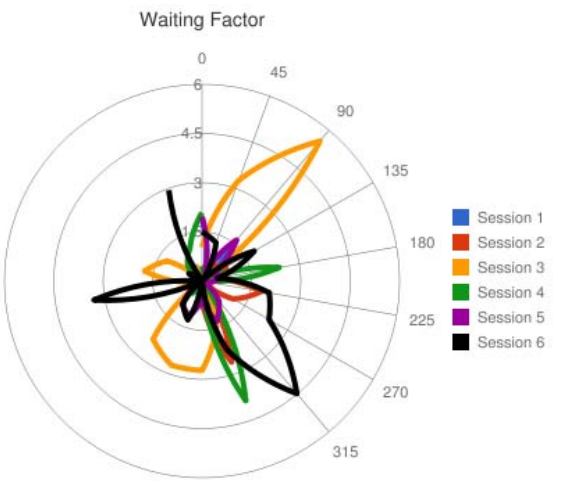

Figure 3. Waiting factor (W) per group and session

\section{RECOMMENDATIONS FOR IMPROVING THE}

\section{EFFICIENCY OF FEEDBACK PROVISION IN LAB SESSIONS}

Based on experimental data, it can be concluded that efficiency problems related to time management are frequent in lab sessions. Some of the causes of these problems are: a) the teacher to student ratio (greater than 20); b) the multiple tasks that the teachers have to carry out (provide feedback, assess, deliver physical artifacts like mobile phones); c) the lack of preparation by the students for the session; and d) the under/over-scripting of the proposed practice, that affects the orchestration of the session.

In this section, a set of actions is proposed in order to leverage the aforementioned problems:

- Usage of problem statements adequately scripted, taking into account the negative effects of overscripting explained in [12]

- Definition of self-assessment mechanisms to encourage students to complete preparatory work.

- Provide the students with the opportunity to verbalize and write down their doubts, as a reflection mechanism (which usually helps them to find the solution themselves and avoid trivial questions).

- Integration of assessment activities in the problem statement, in order to leverage the teacher of this task partially, since he/she may want to avoid cheating by making some related oral questions too.

While these proposals would undoubtedly attack the deeper causes of detected malfunctions, they are not always feasible. A complementary line of action relies on improving the time management and interactions themselves. This work focuses on this line of action and consequently the Class-on tool is proposed for supporting the interactions and orchestration based on the above discussed metrics and learning analytics [2].

Class-on combines a teacher application, a student application and a server framework to keep track of students' activity, progress and demands for help; to provide awareness; and to provide a communication back-channel to optimize teacher intervention while minimizing disruption of students' attention.

The student application records the students' interactions in a non-intrusive way by means of web-based sensors in the problem statements. The problem statement also includes a graphical interface for the students to ask for help to the teacher, and therefore these requests are also tracked. When demanding help, the students will be required to write down their problem and estimate the tutoring time needed (for the scheduling algorithms). This interface is also used for making the students aware of some parameters of the session, for example, the students' progress compared to the average of all the groups. As future work, it is planned to implement delivery mechanisms to track how students interact with pre-, in- and post-class exercises, and also automatic feedback provision for self-assessment activities (e.g., MCQ). The integration of a summative assessment system is also planned as future work.

The teacher application provides awareness about the dynamics in the session. A first version of the user interface was presented in [2] (shown in Figure 4. ), and it has been developed for a portable device (e.g., a tablet) that the teacher could carry easily around the classroom. This interface shows contextualized information about:

- the time the students asked for help

- the tutoring time the students have already consumed

- the progress of each group

- the group being attended by the teacher

- the next group to be helped

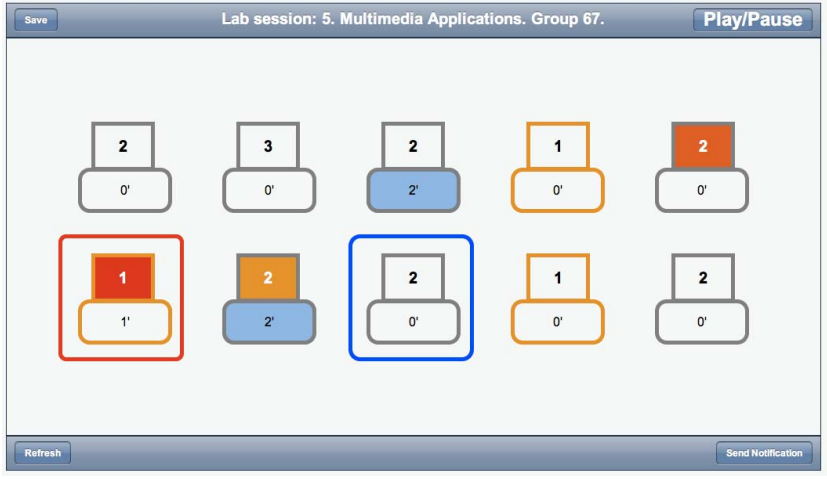

Figure 4. Teacher interface of the Class-on system

This application allows the teacher to:

- indicate tutoring time the teacher is delivering, by simply touching the group to be assisted

- $\quad$ read the issues beforehand, to identify questions that could be solved simply by reading the problem statement or frequent ones that demand a general explanation for the class

- $\quad$ send notifications to all the students [2]

Once completed the session, the application permits the teacher to replay the session and consult a set parameters in order to improve future enactments. If the students' progress during the sessions has to be assessed, the teacher can make use of these parameters for the corresponding grades. 
The described system is originally aimed at supporting lab sessions, where students work on computers so that their activity can be tracked and the teaching staff provides support for them to complete the lab assignments. However, the system scope goes beyond the face-to-face environment, directly supporting blended scenarios too. Mechanisms for tracking the students' activity outside the classroom are provided too, so that information about the previous work and progress of the students is available to the teacher from the very beginning of the session, without the need for an initial assessment of state. Feedback and support can thus be particularized in consequence, for example taking into account whether the student completed prior required readings. Furthermore, the tool could be easily adapted to completely virtual environments, and even beyond synchronous direct face-to-face interactions.

Both in the teacher and students interfaces, some aspects of interaction design have been taken into account in order to improve the users experience with the tool. For example, how the decisions about the learning path are transferred to the learner by means of interaction design in [13].

Finally, the server layer is logs all the events that are tracked before, during and after the class. As the learning materials are typically distributed as web pages, this server layer has been implemented using web technologies, including websockets, nodejs server and mongodb database.

\section{CONCLUSIONS AND FUTURE WORK}

In this work, empirical data have been collected and a set of metrics have been defined for characterizing lab sessions from an efficiency perspective. A case study is discussed with the results of such metrics for a set of typical lab sessions. The empirical data are expected to be further used as a framework for future experimental evaluation.

A supporting tool for improving the feedback provision and interactions in lab sessions based on learning analytics has then been presented. This tool integrates the solutions to the several efficiency problems detected in the orchestration of the sessions:

- providing awareness mechanisms for the teacher for improving the orchestration, distribution of feedback and support, and efficiency of the session;

- providing awareness mechanisms for the students for monitoring their progress and support self-reflection;

- leveraging the labor of the teacher facilitating the identification of trivial or general questions;

- collecting evidences for summative assessment;

- facilitating the analysis of the session for improving future enactments of the learning design.

As future work, the first and most important step is the empirical evaluation of the proposed tool. Additionally:

- Definition of gamification strategies based on the collected data used in order to engage the students.

- Definition of peer support strategies (e.g., indicate that a group could help another one that is progressing slower; or that 2 groups with a shared problem work collaboratively).
- Provide awareness of groups sharing a problem, for the teacher to avoid repeating explanations.

- Test strategies to schedule the teacher interaction (recommendation), e.g., FIFO, Round-Robin, etc.

- Apply a social approach to the questions posed by the students, voting shared popular problems.

- Collect more student interactions and define the corresponding metrics. E.g. attended sessions, late arrivals, prior study of the problem statement, etc.

\section{ACKNOWLEDGMENT}

Research partially supported by the Spanish Plan Nacional de $I+D+I$ projects "Learn3: Towards Learning of the Third Kind" (TIN2008-05163/TSI) and "EEE" (TIN2011-28308-C03-01), and the Madrid regional project "eMadrid: Investigación y desarrollo de tecnologías para el e-learning en la Comunidad de Madrid" (S2009/TIC-1650).

The authors also want to thank teachers and students participating in the experiment, for their disinterested support; and especially to Mar Pérez-Sanagustín for her invaluable insight and ideas related to this contribution.

\section{REFERENCES}

[1] Prince, M. (2004). Does Active Learning Work ? A Review of the Research. Journal of Engineering Education, 93 (July), 223-231.

[2] Gutiérrez Rojas, I., Crespo García, R.M., \& Delgado Kloos, C. (2011) Orchestration and Feedback in Lab Sessions: Improvements in Quick Feedback Provision. Proc. EC-TEL 2011, LNCS, 6964, 424-429.

[3] Barrows, H. S. (1986). A taxonomy of problem-based learning methods. Medical Education, 20(6), 481-486

[4] Sadler, D. R. (1989). Formative assessment and the design of instructional systems. Instructional Science, 18(2), 119-144.

[5] Winter, J. (2003). The changing prepositions of assessment practice: assessment of, for and as learning. British Educational Research Journal, 29(5), 767-772.

[6] Torrance, H. (2007). Assessment as learning? How the use of explicit learning objectives, assessment criteria and feedback in postsecondary education and training can come to dominate learning. Assessment in Education, 14(3), 281-294.

[7] Dillenbourg, P., Järvelä, S., \& Fischer, F. (2009). The evolution of research on computer-supported collaborative learning: from design to orchestration. Technology-Enhanced Learning, 3-19, Springer.

[8] Watts, M. (2003). The orchestration of learning and teaching methods in science education. Canadian Journal of Science Mathematics and Technology Education, 3(4), 451-464.

[9] Prieto, L. P., Holenko Dlab, M., Gutiérrez, I., Abdulwahed, M. and Balid, W. (2011). Orchestrating technology-enhanced learning: a literature review and a conceptual framework. IJ-TEL, 3(6).

[10] Alavi, H. S., Dillenbourg, P., \& Kaplan, F. (2009). Distributed awareness for class orchestration. Learning in the Synergy of Multiple Disciplines. Proc. EC-TEL, 211-225, Springer.

[11] Blikstein, P. (2011). Using learning analytics to assess students behavior in open - ended programming tasks. Proc. Int. Conf. on Learning Analytics and Knowledge (LAK'11).

[12] Dillenbourg, P. (2002). Over-scripting CSCL : The risks of blending collaborative learning with instructional design. P.A. Kirschner (Ed.), Three worlds of CSCL: Can we support CSCL?, 61-92, OUNL.

[13] Lee, S., \& Dron, J. (2009). Giving Learners Control through Interaction Design. Journal of Computers in Mathematics and Science Teaching, 28(2). 